\title{
ANAESTHETIC MANAGEMENT FOR TRACHEOSTOMY OF A PATIENT WITH AN UNUSUAL SUBGLOTTIC OBSTRUCTION
}

Yanki D. Shipmo.

1. Associate Professor, Department of Anaesthesiology, Central Referral Hospital Sikkim Manipal Institute of Medical Sciences, Sikkim,

\section{CORRESPONDING AUTHOR:}

Dr Yanki D. Shipmo,

Dept. of Anaesthesiology, Central Referral Hospital,

Sikkim Manipal Institute of Medical Sciences,

5thMile, Tadong,

Gangtok, Sikkim 737102.

E-mail: ydshipmo2@yahoo.com

ABSTRACT: Airway management, ensuring uninterrupted oxygenation and ventilation, is a fundamental part of the practice of anesthesia and of emergency and critical care medicine. Endotracheal intubation is an airway management technique indicated in a variety of clinical situations, most commonly for the maintenance of the upper airway during general anesthesia, but also in any situation involving the maintenance and protection of the upper airway when the airway may be compromised (8).This case report is about the anaesthetic management of a young adult who presented with acute respiratory distress and stridor. The radiological report was that of soft tissue swelling extending from supraglottic to subglottic region. It was decided to do tracheostomy to relieve his distress but it became a challenging anaesthetic procedure, since local anaesthesia was not possible and general anaesthesia had to be instituted (6). The general anaesthesia was given very cautiously by inducing with Halothane initially and then slowly continued with titrated doses of propofol, followed by intubation with the help of suxamethonium since with gentle ventilation(3), it was possible to aerate the lungs; but far more challenging was with the airway maintenance. Eventually, the tracheostomy and anaesthesia were carried out successfully without any adverse effects, in spite of the challenge of holding onto the ETT so as to prevent the tube from getting dislodged and losing the airway in the process (2).

KEY WORDS: Intubation challenge, Subglottic stenosis

INTRODUCTION: An acute upper airway obstruction is an emergency and it needs to be relieved immediately (8).

The causes of adult tracheal stenosis include trauma, chronic inflammatory diseases, benign neoplasm, malignant neoplasm and collagen vascular diseases (6). Symptoms of upper airway obstruction vary depending on the cause (1), but some symptoms are common to all types of airway blockage. They include agitation, cyanosis, changes in sensorium, confusion and unusual breathing sounds indicating difficulty in breathing. Prompt treatment is often successful; however, the condition is dangerous and may be fatal, even if treated.

Inability to relieve the obstruction can cause progressive deterioration resulting in further respiratory distress, inability to speak /cough making the patient anxious/agitated, with vigorous 


\section{CASE REPORT}

attempts at respiration, causing more distress which can lead to brain damage, respiratory failure and eventually death(8).

It is essential to have a plan of management which includes alternative approaches, should the initial method fail.

CASE REPORT: A 24 year old young man presented in our casualty department with 2 day old history of breathlessness and neck pain. His medical history indicated that he was diagnosed as having pulmonary tuberculosis since last 4 months for which he was taking anti-tuberculosis treatment. However, since the last 2 days he had stopped taking the medication as he could not swallow anything because of the breathing difficulty. The severity of his dyspnoea was such that he was more comfortable sitting up than lying down. There were no obvious signs of any external swelling nor was he febrile. His vital parameters were within normal limits. Arterial Blood Gas analysis could not be done at that time but clinically there were no signs of $\mathrm{CO} 2$ build up Investigation done on the day were :

CBC:....Hb-11.2gm\% ; TLC-5,000 (N66.6\%,L22\%,M10.5\%,E8\%,B0.2\%) Urea-36 ; Creatinine1.0; FBS-81; ECG- wnl.

CXR....B/L mild pleural effusion (costophrenic angles obliterated), patchy opacities in the right lower zone; Heart....within normal limits.

X-ray neck (lat.)...Diffuse laryngeal soft tissue swelling, extending from para-epiglottic fold upto subglottic region: prevertebral space at C6-7 appeared normal.

All other systems were found to be within normal limits and his chest auscultation indicated scattered crepitations. Subsequently he was scheduled for tracheostomy. In the Operation theatre, the ENT surgeons found difficulty in carrying out the tracheostomy under local anaesthesia as the patient could not be made to lie down or position himself in a way that the surgeons could carry out the procedure comfortably and confidently (6).

It was then decided to carry out the procedure under general anaesthesia,

ANAESTHETIC PROCEDURE: The patient was made to lie down as far back as possible, Oxygen by face mask was delivered at 5 litres per minute for 5 minutes, then induction was done with Halothane followed by Inj. Propofol, titrated in $20 \mathrm{mg}$ bolus doses, upto 4 such bolus doses ( total of $80 \mathrm{mg}$ ) till the patient began to lose consciousness. He was made to lie supine with a little head-up tilt, while making sure that he did not suffer further respiratory difficulty. Gentle ventilation allowed adequate aeration of the lungs without causing any distress to the patient. Seeing that one could ventilate without distressing the patient, it was decided to intubate with the help of suxamethonium. After the administration of $60 \mathrm{mg}$ of suxamethonium, cautious ventilation was continued for a full minute.

Once the patient was fully unconscious and fully relaxed, laryngoscopy was done and found the larynx and surrounding tissues to be inflammed and a small cystic swelling was seen at the oesophageal inlet, just behind the larynx. Laryngeal inlet and the vocal cords could be visualized. Intubation was attempted with Size 6.0 cuffed ETT which could not be negotiated beyond the laryngeal inlet. Finally size 5.0 cuffed ETT could be introduced but could not be negotiated beyond the laryngeal inlet as some sort of obstruction was encountered which could not be bypassed; the ETT was forced further down as much as possible with the help of a Magill's forceps and held at that 
point at about $16-17 \mathrm{~cm}$ length and anaesthesia was maintained with $\mathrm{N} 2 \mathrm{O}+\mathrm{O} 2+\mathrm{Halothane}$ and Atracurium . 02saturation was $100 \%$ and EtCO2 was 36mmHg. The surgeons were asked to perform the procedure as quickly as possible. The tracheostomy was done and the airway was secured and a cuffed tracheostomy tube was introduced, through which the anaesthetic procedure was then safely carried out in order for the surgeons to investigate the cause of the upper-airway obstruction.

On laryngoscopy with the rigid laryngoscope, the surgeons found that the larynx was impacted with a hard mass which could not be negotiated to assess the patency of the airway; the description of the larynx was that of a moth- eaten appearance, with severe laryngeal oedema.

The oesophageal bulge was incised and was found to contain clot and mucoid material, which was sent for C/S and Zeil-Nelson Stain because of his history of pulmonary tuberculosis .

The anaesthetic procedure was uneventful and was successfully carried out, although a little precariously. The patient was thus relieved of his immediate respiratory distress. In the subsequent days, upper aero digestive tract endoscopy was done and it was found that the subglottis was narrowed with thickened tissue and the scope could not be negotiated beyond the glottis, even with the rigid bronchoscope. Tissues from the mass were taken and sent for histopathological examination.

DISCUSSION: Partial airway obstruction should be aggressively managed, and immediate preparation for treatment should be instituted under good local anaesthesia or general anaesthesia, which in itself could result in the risk of complete loss of airway control and spontaneous breathing (8). In a normal conscious individual the airway is maintained by the tone of the neck musculature however, the tone is lost under general anaesthesia and therefore an anaesthetized patient is vulnerable to respiratory obstruction and particularly in this patient with a compromised airway, the danger was to make a bad situation into a worse one. Vigorous attempts at laryngoscopy and intubation could result in a traumatic procedure which may also lead to a worsening situation, with bleeding and oedema of the airway creating a situation of 'can't intubate, can't ventilate' (6). For an object stuck in the throat, a laryngoscope/bronchoscope is usually used to remove it but in some cases, there is a requirement for surgical procedure to make an opening in the upper airway below the obstruction, either as a temporary relief to buy time by doing a Cricothyrotomy or a more permanent of solution of Tracheostomy.

In this patient, there was no alternative but to administer General anaesthesia for tracheostomy and it had to be done in a very cautious manner in order to prevent any risk of causing further distress to the patient or even losing the patient in the act. The Endotracheal tube had to be held in place because of the fear of losing the airway while trying to fix it, as there was no other way to maintain the ventilation and the airway control(2).

Usha Rani Nimmagadda et.al described a similar situation "After several attempts, a 5.5-mm (ID) cuffed tracheal tube was passed just beyond the vocal cords, and held in place to prevent dislodgment of the tube"(2); however, in their case, general anaesthesia was instituted only after dilatation of the trachea.

Several other authors have cited similar scenario ; Naveengopal Venugopal et.al had used uncuffed Portex $4.0 \mathrm{~mm}$ ID preformed tube and Magill's forceps was used to assist in guiding the tube beyond the stenotic region (4). 
In this case report, the apprehension was with the fear of dislodgement of the tube and loss of the airway while the patient was already under anaesthesia.. Some authors have cited the use of inhalational induction and spraying of local anaesthetics of the vocal cords but in our scenario, the patient was far to distressed to allow us to spray his larynx and to do any further instrumentation in his airway. We induced with inhalational agent but continued with the cautious doses of propofol and since gentle ventilation could be carried out, the intubation was done with Suxamethonium (3). The key to successful management of the patients with critical tracheal stenosis is to establish safe and efficient gas exchange as rapidly as possible (6). We found that one could deliver safe anaesthesia as long as one could somehow take control of the airway, however risky it maybe.

\section{REFERENCES:}

1. Ashiku SK. Idiopathic laryngotracheal stenosis. Chest Surg Clin North Am 2003; 13:257

2. Usha Rani Nimmagadda, MD, M. Ramez Salem, MD, Michael Friedman, MD, Acute Management of Unsuspected Subglottic Stenosis by Tracheal Dilation Anesth Analg 1995; 80:841-3

3. BA Bulbulia, Ahmed R. Anaesthesia and subglottic airway obstruction. S Afr J Anaesth Analg 2011; 17(2):182-4.

4. N. Venugopal, M. Youssef, S.A. Nortcliffe: Airway management in a case of critical subglottic stenosis: The use of a preformed tracheal tube. The Internet Journal of Anesthesiology 2008; 15(2).DOI: 10.5580/213d

5. Mahfouz AK, Rashid M, Reddy PAl. Anaesthetic management of congenital laryngeal web presenting with acute upper airway obstruction. Anaesthesiol 2010;20(5):719-1

6. Anwar-ul-huda, Muhammad Qamar-ul-Hoda, Sohail Awan. Emergency airway management of a patient with tracheal stenosis. www.jpma.org.pk/full_article_text.php?article_id=2303(September2010)

7. Standley TD, Smith HL. Emergency tracheal catheterization for jet ventilation: a role for the ENT surgeon? J.Larygol.Otol.2005;119(3):235

8. Management of Unanticipated Difficult Intubation Pa Patient Saf Advis 2010;7(4):113 22.(Pennsylvania Patient Safety Advisory Board) 


\section{CASE REPORT}

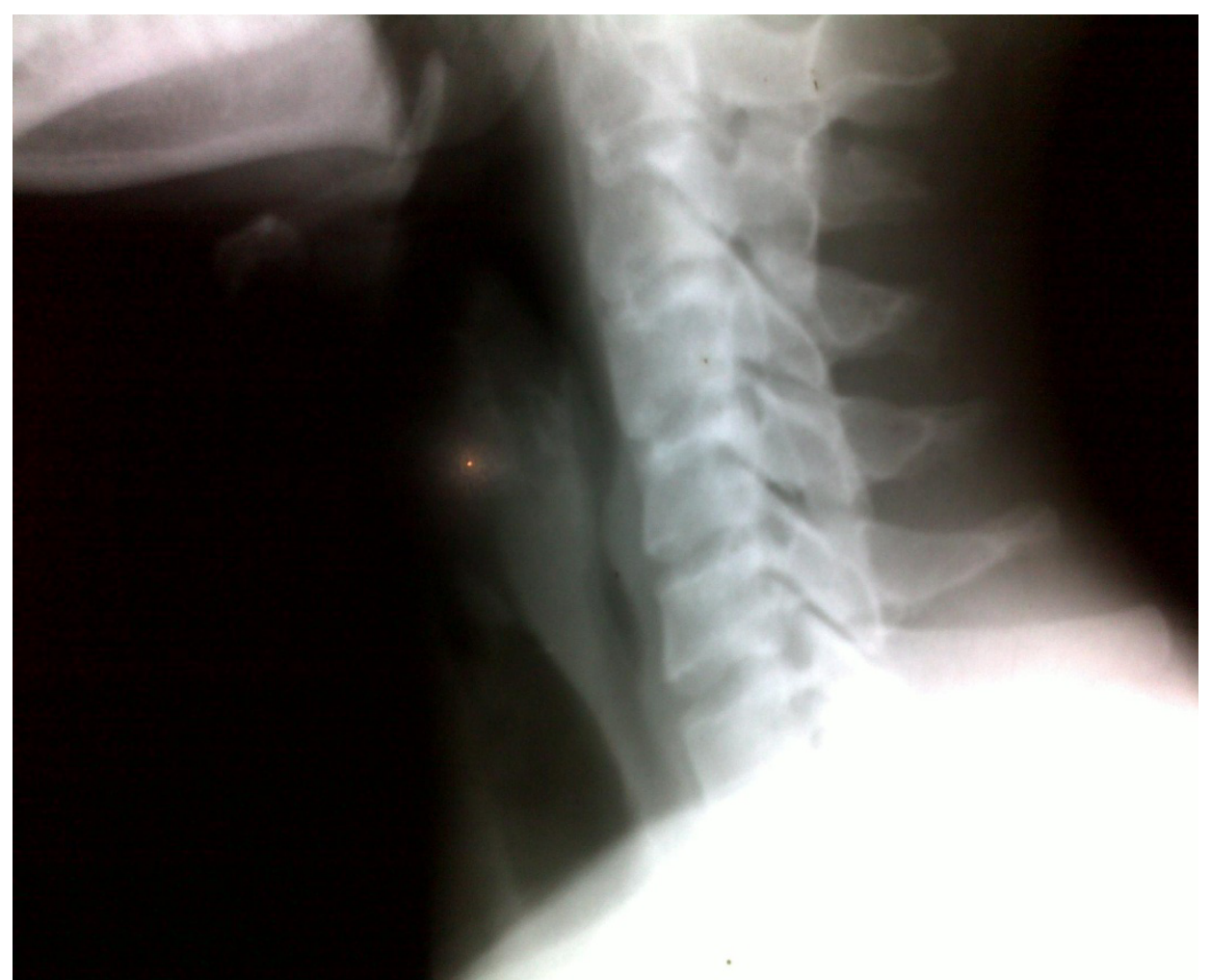

Subglottic obstruction; X-Ray neck (lat .view) 